\title{
Optical Potential and Removal Energies in Lepton Nucleus Scattering
}

\author{
Arie Bodek ${ }^{* \dagger}$ \\ Department of Physics and Astronomy, University of Rochester, Rochester, NY 14627 USA \\ E-mail: Bodek@pas.rochester.edu

\section{Tejin Cai} \\ Department of Physics and Astronomy, University of Rochester, Rochester, NY 14627 USA \\ E-mail: tcai3eur.rochester.edu
}

\begin{abstract}
We summarize some of the results presented in arXiv:1801.07975 [nucl-th][1](to be published in EPJC in 2018) on modeling electron and neutrino QE scattering on a variety of nuclei within the impulse approximation. We find that with three parameters we can describe the final state lepton energy for all of available electron QE data on Lithium, Carbon+Oxygen, Aluminum, Calcium+Argon, Iron and Lead+Gold. The first parameter, the removal energy $\varepsilon^{P, N}$ is extracted from exclusive ee'p spectral function data. The second parameter $V_{e f f}$, which accounts for the interaction of final state leptons and protons with the Coulomb potential of the nucleus, is available from published comparisons of inclusive QE electron and positron cross section. We extract the third parameter $U_{F S I}\left(\vec{q}_{3}^{2}\right)$, which accounts for the interaction of the final state nucleon with the optical potential of the spectator nucleus (FSI), by fitting all available inclusive QE cross sections on nuclear targets. Here $q_{3}$ is the three momentum transfer. With these three parameters we can model the energy of final state electrons and nucleons for all available electron QE scattering data. At present the uncertainty in the value of the removal energy parameters is a the largest source of systematic error in the extraction of the neutrino oscillation parameter $\Delta m^{2}$. The use of the updated parameters in neutrino Monte Carlo generators reduces the systematic uncertainty in the combined removal energy (with FSI corrections) from $\pm 20 \mathrm{MeV}$ to $\pm 5 \mathrm{MeV}$. In this short contribution we only summarize the results for Carbon+Oxygen and Calcium+Argon.
\end{abstract}

The 20th International Workshop on Neutrinos (NuFact2018)

12-18 August 2018

Blacksburg, Virginia

*Speaker.

${ }^{\dagger}$ Supported by the US Department of Energy. 
We summarize some of the results[1] presented in arXiv:1801.07975 [nucl-th] (Removal Energies and Final State Interaction in Lepton Nucleus Scattering" to be published in EPJC in 2018) on modeling electron and neutrino quasielastic (QE) scattering on a variety of nuclei within the impulse approximation. Fig. 1 shows the $1 \mathrm{p} 1 \mathrm{~h}$ process for electron (left) and neutrino (right) QE scattering from an off-shell bound nucleon of momentum $\vec{p}_{i}=\vec{k}$ in a nucleus of mass A. Here, the nucleon is moving in the mean field (MF) of all the other nucleons in the nucleus. The on-shell recoil excited $[A-1]^{*}$ spectator nucleus has a momentum $\vec{p}_{(A-1) *}=-\vec{k}$ and a mean excitation energy $\left\langle E_{x}^{P, N}\right\rangle$. The off-shell energy of the interacting nucleon is $E_{i}=M_{A}-\sqrt{\left(M_{A-1} *\right)^{2}+\vec{k}^{2}}=$ $M_{A}-\sqrt{\left(M_{A-1}+E_{x}^{P, N}\right)^{2}+\vec{k}^{2}}=M_{P}-\varepsilon^{P, N}$, where $\varepsilon^{P}=S^{P, N}+\left\langle E_{x}^{P, N}\right\rangle+\frac{\vec{k}^{2}}{2 M_{A-1}^{*}}$. Here $S^{P, N}$ the separation energy needed to separate a nucleon from the nucleus.

Current neutrino MC generators (e.g. GENIE) do not include the effect of the excitation of the spectator nucleus, nor do they include the effects of the interaction of the final state leptons and nucleons with the optical and Coulomb potential of the nucleus. We extract the mean excitation energy $\left\langle E_{x}^{P}\right\rangle$ from exclusive $e e^{\prime} P$ spectral function measurements. We include the effects of the interaction of the final state leptons and protons with Coulomb field of the nucleus by using published parameters $V_{\text {eff }}$ obtained from a comparison of electron and positron QE differential cross sections [2] , and model the effect of the interaction of final state nucleons with the optical potential of the nucleus another parameter with $\left|U_{F S I}\left(\vec{q}_{3}^{2}\right)\right|$. We set energy of a proton in the final state to $E_{f}^{P}=\sqrt{\left(\vec{k}+\vec{q}_{3}\right)^{2}+M_{P}^{2}}-\left|U_{F S I}\left(\vec{q}_{3}^{2}\right)\right|+\left|V_{e f f}^{P}\right|$, and extract $\left|U_{F S I}\left(\vec{q}_{3}^{2}\right)\right|$ from a comparison of the relativistic Fermi gas (RFG) model to measurements of inclusive QE e-A differential cross sections. The data samples include: four ${ }_{3}^{6} \mathrm{Li}$ spectra, $33{ }_{6}^{12} \mathrm{C}$ spectra, five ${ }_{8}^{16} \mathrm{O}$ spectra, seven ${ }_{18}^{27} \mathrm{Al}$

Electron scattering on proton

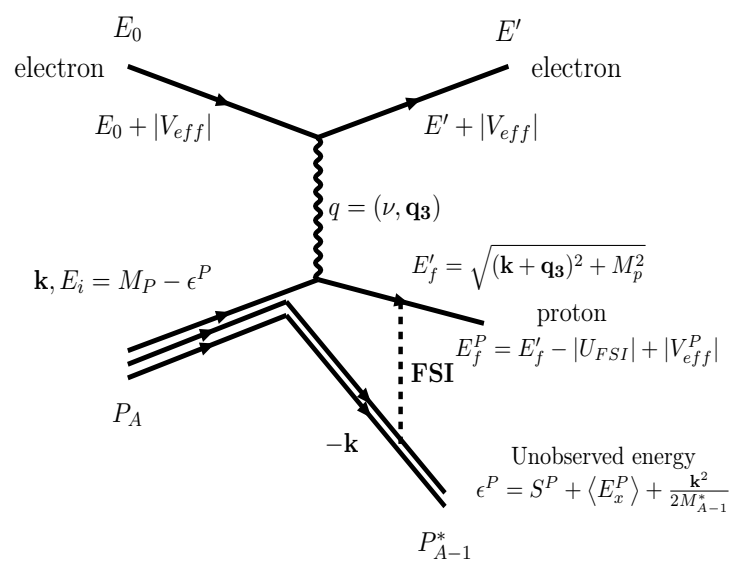

Neutrino scattering on neutron

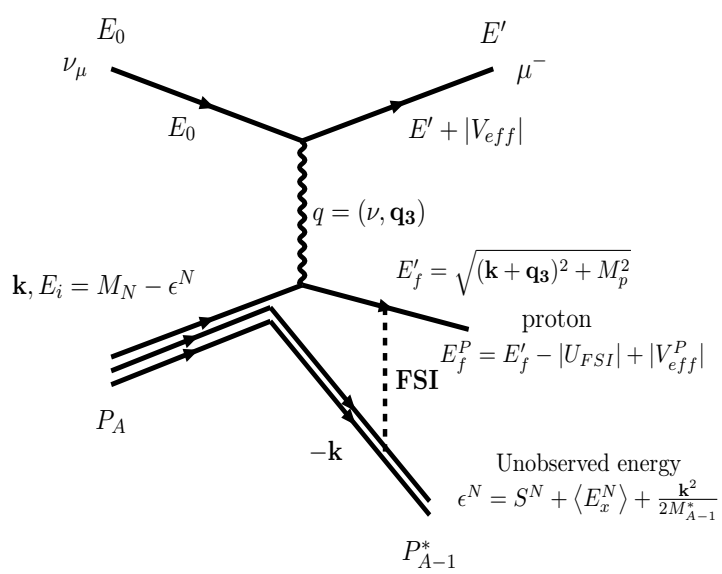

Figure 1: Electron (Left) and neutrino (right) $\mathrm{QE}$ scattering from an off-shell bound proton.

spectra, $29{ }_{20}^{40} \mathrm{Ca}$ spectra, two ${ }_{18}^{40} \mathrm{Ar}$ spectra, $30{ }_{26}^{56} \mathrm{Fe}$ spectra, $23{ }_{82}^{208} \mathrm{~Pb}$ spectra and one ${ }_{79}^{197} \mathrm{Au}$ spectrum. Most of the QE differential cross sections are available on the $\mathrm{QE}$ electron scattering archive[3]. Figure 2, shows examples of three of 33 fits to QE differential cross sections for ${ }_{6}^{12} \mathrm{C}$. The solid blue curve is the RFG fit with the best value of $U_{F S I}$. The black dashed curve is a simple parabolic fit used to estimate the systematic error. The red dashed curve is the RFG model with $U_{F S I}=V_{\text {eff }}=0$. The extracted values of $U_{F S I}\left(\vec{q}_{3}^{2}\right)$ versus $\vec{q}_{3}^{2}$ for ${ }_{6}^{12} \mathrm{C}+{ }_{8}^{16} \mathrm{O}$, and ${ }_{20}^{40} \mathrm{Ca}+{ }_{18}^{40} \mathrm{Ar}$ are shown in Figure 3. We fit the extracted values of $U_{F S I}\left(\vec{q}_{3}^{2}\right)$ versus $\vec{q}_{3}^{2}$ for $\overrightarrow{q_{3}^{2}}>0.1 \mathrm{GeV}^{2}$ to a linear 
function. The intercepts at $\vec{q}_{3}^{2}=0$ and the slopes of $U_{F S I}\left(\vec{q}_{3}^{2}\right)$ are shown on the Figure. We only fit
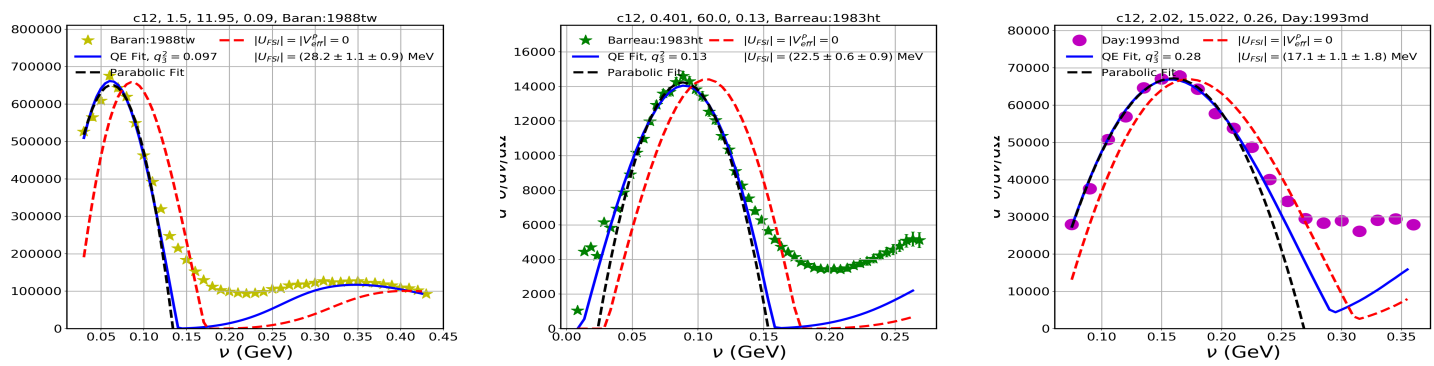

Figure 2: Examples of fits for three out of 33 carbon $\left({ }_{6}^{12} \mathrm{C}\right) \mathrm{QE}$ differential cross sections. The solid blue curve is the RFG fit with the best value of $U_{F S I}$. The black dashed curve is the simple parabolic fit used to estimate the systematic error. The red dashed curve is the RFG model with $U_{F S I}=V_{e f f}=0$.

to the data in the top $1 / 3$ of the QE distribution and extract the best value of $U_{F S I}\left(\vec{q}_{3}^{2}\right)$. In the fit we let the normalization of the QE peak float to agree with data. The systematic error is estimated by using the difference between $v_{\text {peak }}^{\text {parabola }}$ and $v_{\text {peak }}^{r f g}$ as a systematic error in our extraction of $U_{F S I}\left(\vec{q}_{3}^{2}\right)$. Here $\vec{q}_{3}$ is evaluated at the peak of the QE distribution. With the three parameters we can model the energy of final state electrons and nucleons for all available electron QE scattering data. With the updated parameters the systematic uncertainty in the combined removal energy (with FSI corrections) in neutrino MC generators is reduced from $\pm 20 \mathrm{MeV}$ to $\pm 5 \mathrm{MeV}$.
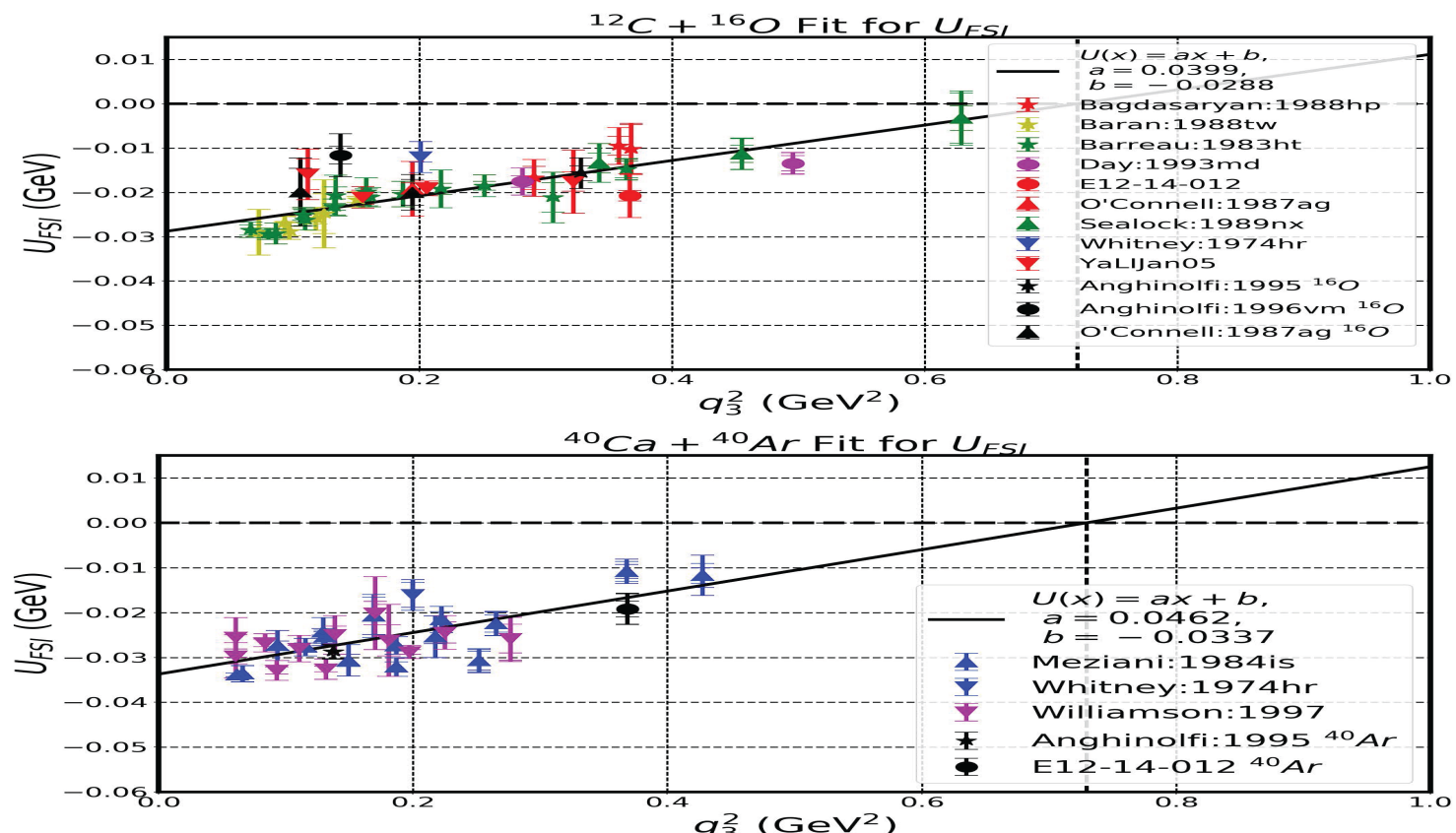

Figure 3: Top plot: Extracted values of $U_{F S I}$ versus $\vec{q}_{3}^{2}$ for 33 Carbon $\left({ }_{6}^{12} \mathrm{C}\right)$ and four Oxygen $\left({ }_{8}^{16} \mathrm{O}\right)$ spectra. Bottom plot: Extracted values of $U_{F S I}$ versus $\vec{q}_{3}^{2}$ for 29 Calcium ${ }_{20}^{40} \mathrm{Ca}$ ) and two Argon ( $\left({ }_{18}^{40} \mathrm{Ar}\right)$ spectra.

\section{References}

[1] "Removal Energies and Final State Interaction in Lepton Nucleus Scattering", Arie Bodek and Tejin Cai, arXiv:1801.07975 [nucl-th] (to be published in EPJC 2018).

[2] P. Gueye et al. Phys. Rev. C60, 044308 (1999).

[3] Quasielastic Electron Nucleus Scattering Archive: http://faculty.virginia.edu/qes-archive/. 